\title{
ACMG statement on noninvasive prenatal screening for fetal aneuploidy
}

\author{
Anthony R. Gregg, MD1, S.J. Gross, MD², R.G. Best, PhD³, K.G. Monaghan, PhD4, K. Bajaj, MD², \\ B.G. Skotko, MD ${ }^{5}$, B.H. Thompson, $\mathrm{MD}^{6}$ and M.S. Watson, $\mathrm{PhD}^{6}$; are The Noninvasive Prenatal \\ Screening Work Group of the American College of Medical Genetics and Genomics
}

\begin{abstract}
Disclaimer: This statement is designed primarily as an educational resource for clinicians to help them provide quality medical services. Adherence to this statement is completely voluntary and does not necessarily assure a successful medical outcome. This statement should not be considered inclusive of all proper procedures and tests or exclusive of other procedures and tests that are reasonably directed to obtaining the same results. In determining the propriety of any specific procedure or test, the clinician should apply his or her own professional judgment to the specific clinical circumstances presented by the individual patient or specimen. Clinicians are encouraged to document the reasons for the use of a particular procedure or test, whether or not it is in conformance with this statement. Clinicians also are advised to take notice of the date this statement was adopted, and to consider other medical and scientific information that becomes available after that date. It also would be prudent to consider whether intellectual property interests may restrict the performance of certain tests and other procedures.
\end{abstract}

Noninvasive assessment of the fetal genome is now possible using next-generation sequencing technologies. The isolation of fetal DNA fragments from maternal circulation in sufficient quantity and sizes, together with proprietary bioinformatics tools, now allows patients the option of noninvasive fetal aneuploidy screening. However, obstetric care providers must become familiar with the advantages and disadvantages of the utilization of this approach as analysis of cell-free fetal DNA moves into clinical practice. Once informed, clinicians can provide efficient pretest and posttest counseling with the goal of avoiding patient harm. It is in the public's best interest that test

The American College of Medical Genetics and Genomics (ACMG) believes that the application of genetic technology, particularly when used in the prenatal setting, needs to be supported by prospective clinical trials and considered carefully before its incorporation into routine clinical care. The ACMG has previously published guidelines on prenatal screening for Down syndrome, which have successfully assisted health-care providers and their patients during pregnancy. ${ }^{1}$

One of the major breakthroughs in obstetrical care was the advent of prenatal genetic diagnosis, initially by amniocentesis in the second trimester of pregnancy. Subsequently, chorionic villus sampling during the first trimester allowed for earlier diagnosis and management. However, the potential risk of fetal loss secondary to an invasive procedure has driven the search for noninvasive approaches for genetic screening and diagnosis. Until recently, noninvasive screening for aneuploidy relied on either the measurement of maternal serum analytes and/or ultrasonography with positive screen rates of $\sim 5 \%$ and detection rates of $50-95 \%$, depending on the screening strategy results contain key elements and that laboratories adhere to established quality control and proficiency testing standards. The analysis of cell-free fetal DNA in maternal circulation for fetal aneuploidy screening is likely the first of major steps toward the eventual application of whole fetal genome/whole fetal exome sequencing.

Genet Med 2013:15(5):395-398

Key Words: cell-free fetal DNA; noninvasive prenatal testing; prenatal genetic screening

utilized. More recent advances in genomics and genomic technologies have resulted in the development of a noninvasive prenatal screening (NIPS) test using cell-free fetal DNA sequences isolated from a maternal blood sample..$^{2-6}$ About $10 \%$ of DNA in maternal serum is of fetal origin; ${ }^{4,7,8}$ this has been used for prenatal $\mathrm{Rh}$ determination and gender identification. Using nextgeneration sequencing platforms, millions of amplified genetic fragments can be sequenced in parallel (massively parallel sequencing). Platforms differ according to whether amplified regions throughout the genome, chromosome-specific regions, or single-nucleotide polymorphisms are the targets for sequencing. Furthermore, by using powerful bioinformatics tools, differences between maternal and fetal sequences and dosage differences in identical sequences or a reference chromosome can be determined and used for noninvasive screening for fetal aneuploidy. ${ }^{9,10}$

Although studies are promising and demonstrate high sensitivity and specificity with low false-positive rates, there are limitations to NIPS. Specificity and sensitivity are not uniform for

${ }^{1}$ Department of Obstetrics and Gynecology, University of Florida, Gainesville, Florida, USA; ${ }^{2}$ Department of Obstetrics and Gynecology, Albert Einstein College of Medicine, Bronx, New York, USA; ${ }^{3}$ University of South Carolina School of Medicine, Greenville, South Carolina, USA; ${ }^{4}$ Department of Medical Genetics, Henry Ford Health System, Detroit, Michigan, USA; ${ }^{5}$ Division of Medical Genetics, Massachusetts General Hospital, Boston, Massachusetts, USA; ${ }^{6}$ American College of Medical Genetics and Genomics, Bethesda, Maryland, USA. Correspondence: Anthony R. Gregg (greggar@ufl.edu) 
all chromosomes; this is due, at least in part, to differing content of cytosine and guanine nucleotide pairs. ${ }^{9}$ False-positive screening results do occur. Furthermore, the sequences derived from NIPS are derived from the placenta and therefore, like chorionic villus sampling, may not reflect the true fetal karyotype. Therefore, invasive testing is recommended for confirmation of a positive screening test and should remain an option for patients seeking a definitive diagnosis. This document addresses some of the challenges of incorporating NIPS for fetal aneuploidy into obstetrical practice.

\section{WHERE DOES NIPS FIT INTO THE ANEUPLOIDY SCREENING PARADIGM?}

NIPS is, as the acronym implies, a screening test to identify pregnancies at risk for common autosomal aneuploidies (e.g., trisomy 21,18 , and 13). ${ }^{6}$ Some laboratories also offer screening for sex chromosome aneuploidies.

For women seeking a definitive diagnosis, invasive procedures for diagnostic testing, such as amniocentesis or chorionic villus sampling, should be offered.

\section{WHAT ARE THE CURRENT LIMITATIONS OF NIPS?}

1. Risk assessment is limited to specific fetal aneuploidies (trisomy 13, 18, and 21) at this time. Some platforms also screen for sex chromosome abnormalities. Approximately 50\% of cytogenetic abnormalities routinely identified by amniocentesis will not be detected when trisomy 21,18 , and 13 are the only aneuploidies being screened. When patients $<35$ years or $>35$ years are considered separately, 75 and $43 \%$ of cytogenetic abnormalities will be missed, respectively. ${ }^{11,12}$

2. Chromosomal abnormalities such as unbalanced translocations, deletions, and duplications will not be detected by NIPS. Therefore, when fetal anomalies are detected, invasive diagnostic testing and cytogenomic microarray analysis are more likely to detect chromosomal imbalances than NIPS and may be a better testing option..$^{13}$

3. NIPS is not able to distinguish specific forms of aneuploidy. For example, NIPS cannot determine if Down syndrome is due to the presence of an extra chromosome (trisomy 21), a Robertsonian translocation involving chromosome 21, or high-level mosaicism. Identification of the mechanism of aneuploidy is important for recurrence risk counseling and emphasizes the importance of diagnostic testing following NIPS.

4. NIPS does not screen for single-gene mutations.

5. Uninformative test results due to insufficient isolation of cell-free fetal DNA could lead to a delay in diagnosis or eliminate the availability of information for risk assessment. Biologic factors associated with reduced available cell-free fetal DNA include a high body mass index and early gestational age $(<10 \text { weeks gestation) })^{14,15}$

6. Currently, it takes longer for NIPS test results to be returned than for test results on maternal serum analytes. Providers should keep this in mind when offering patients NIPS if timing is important for reproductive decision making. In most cases, NIPS is offered between 10 and 20 weeks gestation, which allows time for follow-up of positive test results. It is reasonable to offer NIPS after 20 weeks if an expectant women desires information regarding risk, reassurance, or knowledge in order to inform obstetrical management and/ or preparation for birth.

7. NIPS does not screen for open neural tube defects. Maternal serum $a$-fetoprotein testing should still be offered at 15-20 weeks gestation to screen for open neural tube defects even when NIPS is performed. ${ }^{1}$

8. NIPS does not replace the utility of a first-trimester ultrasound examination, which has been proven to be useful for accurate gestational dating, assessment of the nuchal translucency region to identify a fetus at increased risk for a chromosome abnormality, identification of twins and higher-order pregnancies, placental abnormalities, and congenital anomalies. ${ }^{16-19}$

9. Limited data are currently available on the use of NIPS in twins and higher-order pregnancies. Utilization in these clinical settings may depend on specific laboratory platforms, proprietary bioinformatics, and clinical validation studies.

10. NIPS has no role in predicting late-pregnancy complications.

\section{SHOULD PRETEST OR POSTTEST GENETIC COUNSELING ABOUT ANEUPLOIDY SCREENING BE PERFORMED?}

Pretest information should be provided by a prenatal care provider, a trained designee, or a genetic counselor to ensure patients make informed decisions. Aneuploidy screening is not a routine prenatal test; it is acceptable for patients to decline screening.

Pretest information should include:

1. A brief explanation of the purpose of NIPS.

2. Advantages of NIPS as compared with maternal serum analyte screening.

- On the basis of available data, detection rates appear to be higher.

- There is a high negative predictive value for Down syndrome. This may be important for patients seeking to avoid the risks (e.g., fetal loss) inherent with invasive testing.

- NIPS has a lower false-positive rate, meaning fewer women will receive a "positive" screen, necessitating fewer invasive procedures.

- Risk assessment is less dependent on gestational age.

3. Considerations for follow-up invasive testing if NIPS indicates an increased risk for aneuploidy.

4. Limitations of NIPS.

Posttest counseling is recommended when NIPS indicates that a patient is at high risk or has a "screen-positive" result. When a "screen-negative" result is encountered, residual 
risk should be reinforced. When obstetric care providers are uncomfortable with providing posttest counseling, referral to a certified genetics professional is warranted. Posttest counseling should be individualized but should include at least the following discussion points:

1. There is a possibility of false-positive screening results, which may be due to confined placental mosaicism or theoretically a "vanishing twin."

2. NIPS is not diagnostic; therefore, confirmatory testing (chorionic villus sampling or amniocentesis) is recommended, and the risks of those procedures should be reviewed.

3. If the patient declines invasive testing, an effort should be made to obtain a sample of cord blood for postnatal confirmation by karyotype or cytogenomic microarray analysis.

4. Accurate, up-to-date, and balanced information about Down syndrome (or other tested conditions) should be provided. There are a number of resources available (see Resources).

Posttest counseling after a "screen-uninformative" result should include the offer of invasive diagnostic testing.

\section{Commentary}

The importance of sensitivity and specificity in comparing clinical tests and the use of these measures in a public health environment cannot be overstated; however, patient care focuses on two distinctly different metrics used to determine the validity of clinical tests: positive and negative predictive value (PPV and NPV, respectively). Clinical sensitivity and specificity are independent of the prevalence of Down syndrome and are known to be high when comparing NIPS technologies with other methods of screening for fetal aneuploidy. However, PPV and NPV can be expected to vary with the population prevalence of Down syndrome. Although the NPV can be considered high, PPV is not as desirable owing to the relatively low prevalence of Down syndrome across the age spectrum of women giving birth (0.6\% at second trimester amniocentesis). ${ }^{11}$ By definition, diagnostic tests, as compared with screening tests, have very high PPV and NPV, approaching 100\%.

\section{WHAT SHOULD LABORATORIES PERFORMING NIPS DISCLOSE WHEN REPORTING RESULTS TO HEALTH-CARE PROVIDERS?}

There are multiple ways to express risk; however, test results should be expressed in the clearest form possible to avoid confusion and misinterpretation. All reports should clearly state that NIPS is a screening test and not diagnostic. The language in the report should clarify the need for posttest counseling for patients with "screen-positive" or "screen-uninformative" results.

\section{WHAT TYPES OF OVERSIGHT ARE REQUIRED OF THE ANALYTICAL AND BIOINFORMATICS ASPECTS OF NIPS TEST SYSTEMS?}

The ACMG recommends compliance with its standards and guidelines for clinical genetics laboratories. Considering the nature of the methods used, NIPS is subject to the same quality control and proficiency testing requirements as those for clinical molecular laboratory tests. Quality control should include the entire test process, including preanalytical, analytical, and postanalytical phases. Until external proficiency testing programs sponsored by a professional or regulatory organization are available, alternative methods for proficiency testing, preferably using an interlaboratory comparison method, is required. Test performance characteristics should be available to patients and providers accessing testing.

NIPS methodologies take advantage of proprietary bioinformatics to determine the risk of specific aneuploidies for a given pregnancy. Comparative effectiveness studies of the performance of the different algorithms should be performed.

\section{CONCLUSION}

NIPS for fetal aneuploidy has arrived; however, as with most new technologies, there is room for refinement. The ACMG encourages providers of NIPS technology to make serious efforts to provide the more clinically relevant metrics-PPV and NPV. This can be accomplished through a funded registry where efforts are made to confirm and archive not only true positives, but also false positives and true negatives. The ethical principle of distributive justice causes us to reflect on who will pay for NIPS and who should be insured for the procedure. No doubt NIPS costs will come down; however, for NIPS to establish roots in the perinatal aneuploidy screening paradigm, cost as a barrier to population-based screening must be minimized. NIPS technology is perhaps only a few steps removed from an eventual whole-genome array, whole-genome sequencing, or whole-exome sequencing of noninvasively isolated cell-free fetal DNA. Whether this best comes about by simultaneously amplifying maternal sequence and subtracting this from fetal sequence, or after isolation and amplification of fetal sequences unique from maternal, is yet to be resolved.

\section{RESOURCES}

\section{Understanding a Down syndrome diagnosis}

This material (http://www.lettercase.org), available in print and digital versions, both in English and in Spanish, is intended for expectant couples who have received a prenatal diagnosis of Down syndrome but have not yet made a decision regarding their pregnancy options. The book was prepared with assistance from the ACMG, the American Congress of Obstetricians and Gynecologists, the National Society of Genetic Counselors, the National Down Syndrome Society, and the National Down Syndrome Congress.

\section{"Brighter Tomorrows"}

This site (for medical professionals: http://www.brighter tomorrows.org; for expectant parents: http://www.brighter tomorrows.org) provides simulation training for health-care professionals who deliver a prenatal diagnosis to expectant couples; the Web page also provides information, in English and Spanish, about Down syndrome to new and expectant couples 
who have received a prenatal diagnosis of Down syndrome. The project was funded by federal grants; efficacy was researched and published in peer-reviewed journals.

\section{"Health-care supervision for children with Down syndrome"} This clinical report (http://pediatrics.aappublications.org/ content/128/2/393), written by the Committee on Genetics of the American Academy of Pediatrics, provides guidance to the health-care professional involved in prenatal consultations; resources for parents are also listed.

\section{ACKNOWLEDGMENTS}

The Work Group thanks Deborah A. Driscoll, MD, Professor and Chair of Obstetrics and Gynecology, University of Pennsylvania Health System, for her critical reading and suggestions that have improved this article.

\section{DISCLOSURE}

S.J.G. discloses grants related to prenatal screening. B.G.S. volunteers in a nonpaid capacity with Down syndrome advocacy organizations. The other authors declare no conflict of interest.

\section{REFERENCES}

1. Driscoll DA, Gross SJ. First trimester diagnosis and screening for fetal aneuploidy. Genet Med 2008; 10:73-75

2. Palomaki GE, Kloza EM, Lambert-Messerlian GM, et al. DNA sequencing of maternal plasma to detect Down syndrome: an international clinical validation study. Genet Med 2011:13:913-920.

3. Ehrich M, Deciu C, Zwiefelhofer T, et al. Noninvasive detection of fetal trisomy 21 by sequencing of DNA in maternal blood: a study in a clinical setting. Am J Obstet Gynecol 2011;204:205.e1-205.11

4. Norton ME, Brar H, Weiss J, et al. Non-Invasive Chromosomal Evaluation (NICE) Study: results of a multicenter prospective cohort study for detection of fetal trisomy 21 and trisomy 18. Am J Obstet Gynecol 2012;207:137.e1-137.e8.

5. Bianchi DW, Platt LD, Goldberg JD, Abuhamad AZ, Sehnert AJ, Rava RP. Genome-wide fetal aneuploidy detection by maternal plasma DNA sequencing. Obstet Gynecol 2012;119:890-901.
6. Nicolaides KH, Syngelaki A, Ashoor G, Birdir C, Touzet G. Noninvasive prenatal testing for fetal trisomies in a routinely screened first-trimester population. Am J Obstet Gynecol 2012;207:374.e1-374.e6.

7. Ashoor G, Syngelaki A, Poon LC, Rezende JC, Nicolaides KH. Fetal fraction in maternal plasma cell-free DNA at 11-13 weeks' gestation: relation to maternal and fetal characteristics. Ultrasound Obstet Gynecol 2013;41: 26-32.

8. Sparks AB, Struble CA, Wang ET, Song K, Oliphant A. Noninvasive prenatal detection and selective analysis of cell-free DNA obtained from maternal blood: evaluation for trisomy 21 and trisomy 18. Am J Obstet Gynecol 2012;206:319. e1-319.e9.

9. Chen EZ, Chiu RW, Sun H, et al. Noninvasive prenatal diagnosis of fetal trisomy 18 and trisomy 13 by maternal plasma DNA sequencing. PLOS ONE 2011;6:e21791

10. Liao GJ, Chan KC, Jiang P, et al. Noninvasive prenatal diagnosis of fetal trisomy 21 by allelic ratio analysis using targeted massively parallel sequencing of maternal plasma DNA. PLoS ONE 2012;7:e38154

11. Forabosco A, Percesepe A, Santucci S. Incidence of non-age-dependent chromosomal abnormalities: a population-based study on 88965 amniocenteses. Eur J Hum Genet 2009;17:897-903.

12. Grati FR, Barlocco A, Grimi B, et al. Chromosome abnormalities investigated by non-invasive prenatal testing account for approximately $50 \%$ of fetal unbalances associated with relevant clinical phenotypes. Am J Med Genet A 2010;152A:1434-1442

13. Wapner RJ, Martin CL, Levy B, et al. Chromosomal microarray versus karyotyping for prenatal diagnosis. N Eng/ J Med 2012;367:2175-2184.

14. Wataganara T, Peter I, Messerlian GM, Borgatta L, Bianchi DW. Inverse correlation between maternal weight and second trimester circulating cell-free fetal DNA levels. Obstet Gynecol 2004;104:545-550.

15. Ashoor G, Poon L, Syngelaki A, Mosimann B, Nicolaides KH. Fetal fraction in maternal plasma cell-free DNA at 11-13 weeks' gestation: effect of maternal and fetal factors. Fetal Diagn Ther 2012;31:237-243.

16. Bennett KA, Crane JM, O'shea P, Lacelle J, Hutchens D, Copel JA. First trimester ultrasound screening is effective in reducing postterm labor induction rates: a randomized controlled trial. Am J Obstet Gynecol 2004;190: 1077-1081.

17. Stenhouse E, Hardwick C, Maharaj S, Webb J, Kelly T, Mackenzie FM Chorionicity determination in twin pregnancies: how accurate are we? Ultrasound Obstet Gynecol 2002;19:350-352.

18. Souka AP, Pilalis A, Kavalakis Y, Kosmas Y, Antsaklis P, Antsaklis A. Assessment of fetal anatomy at the 11-14-week ultrasound examination. Ultrasound Obstet Gynecol 2004;24:730-734.

19. Fong KW, Toi A, Salem S, et al. Detection of fetal structural abnormalities with US during early pregnancy. Radiographics 2004;24:157-174. 